\title{
Molecular mechanisms of drug resistance and its reversal in cancer
}

\author{
Melis Kartal-Yandim ${ }^{1} *$, Aysun Adan-Gokbulut ${ }^{1} *$, and Yusuf Baran ${ }^{1,2}$ \\ ${ }^{1}$ Department of Molecular Biology and Genetics, Izmir Institute of Technology, Urla, Izmir, Turkey and ${ }^{2}$ Faculty of Life and Natural Sciences, \\ Abdullah Gul University, Kayseri, Turkey
}

\begin{abstract}
Chemotherapy is the main strategy for the treatment of cancer. However, the main problem limiting the success of chemotherapy is the development of multidrug resistance. The resistance can be intrinsic or acquired. The resistance phenotype is associated with the tumor cells that gain a cross-resistance to a large range of drugs that are structurally and functionally different. Multidrug resistance arises via many unrelated mechanisms, such as overexpression of energy-dependent efflux proteins, decrease in uptake of the agents, increase or alteration in drug targets, modification of cell cycle checkpoints, inactivation of the agents, compartmentalization of the agents, inhibition of apoptosis and aberrant bioactive sphingolipid metabolism. Exact elucidation of resistance mechanisms and molecular and biochemical approaches to overcome multidrug resistance have been a major goal in cancer research. This review comprises the mechanisms guiding multidrug resistance in cancer chemotherapy and also touches on approaches for reversing the resistance.
\end{abstract}

\section{Keywords}

ABC transporters, apoptosis, bioactive sphingolipids, cancer, cell cycle alteration, multidrug resistance

\section{History}

Received 5 October 2013

Revised 20 December 2014

Accepted 21 December 2014

Published online 11 March 2015

\section{Introduction}

Anticancer drugs with different properties and targets are effectively used in the treatment of cancer (Gottesman, 2002). However, there is no fully effective cancer treatment strategy, especially for certain cancers. A major cause of the treatment failure in cancer patients includes the development of intrinsic or acquired drug resistance against chemotherapeutic agents (Gottesman, 2002; Larsen et al., 2000). Intrinsic resistance could be already present at diagnosis and cancer cells do not respond to standard chemotherapy drugs from the beginning while acquired resistance could be observed after chemotherapy (Giaccone \& Pinedo, 1996). The resistance phenotype is associated with the tumor cells gaining a cross-resistance to large range of drugs with different cellular targets and structures, which is called multiple drug resistance (MDR) (Krishna \& Mayer, 2000). MDR is especially problematic in acquired drug resistance in which the use of high dose drugs to overcome resistance is ineffective and toxic when MDR develops, causing less effective chemotherapy (Liscovitch \& Lavie, 2002). On the other hand, it is also known that MDR could be multifactorial with at least two different resistance mechanisms against the same drug in the same tumor cell. (Larsen \& Skladanowski, 1998). Numerous mechanisms have been described to explain MDR in mammalian cells

\footnotetext{
*These authors have equal contribution to the article.

Address for correspondence: Prof. Dr. Yusuf Baran, Department of Molecular Biology and Genetics, Science Faculty, Izmir Institute of Technology, Urla, 35430 İzmir, Turkey. Tel: +90 232 7507315. Fax: +90 232 7507509. E-mail: yusufbaran@iyte.edu.tr
}

(Figure 1) and they have been grouped into cellular and noncellular mechanisms (Krishna \& Mayer, 2000). Non-cellular mechanisms are characterized by in vivo tumor growth and typically found in solid tumors with unique extracellular properties. (Demant et al., 1990) for instance, the acidic environment due to lactic acid production by hypoxic tumor cells has been thought to be responsible for resistance against some drugs whose uptake is dependent on the $\mathrm{pH}$ gradient across the membranes (Demant et al., 1990). Cellular resistance mechanisms are defined by alterations in the biochemistry of malignant cells and classified into nonclassical MDR phenotypes and transport-based classical MDR phenotypes (Stavrovskaya, 2000). Non-classical MDR describes non-transport based mechanisms and includes altered activity of enzymes such as glutathione S-transferase (GST) and topoisomerase that can decrease the cytotoxic activity of drugs and changes in the balance of proteins involved in apoptosis (Schisselbauer et al., 1990). Transportbased classical MDR mechanisms are related to the ATPbinding cassette $(\mathrm{ABC})$ family of membrane transport ATPases (Krishna \& Mayer, 2000). These transporters can be overexpressed in cancer cells and pump anticancer drugs out of the cell, resulting in a lack of effective concentrations of drugs for therapy (Choi, 2005). In conclusion, the ability of cancer cells to become simultaneously resistant to different drugs remains a significant obstacle to successful chemotherapy although chemotherapeutics are the most effective treatment agents for most types of cancer. Therefore, exact elucidation of resistance mechanisms and various approaches to overcome multidrug resistance have been a major goal in cancer research. In this review, we describe multidrug 
Figure 1. The mechanisms of drug resistance in cancer cells. These mechanisms include removal of drugs by transporters, specific drug metabolism or detoxification, intracellular drug sequestration, changes in the expression of genes involved in apoptosis, altered cell cycle events and aberrant ceramide metabolism.

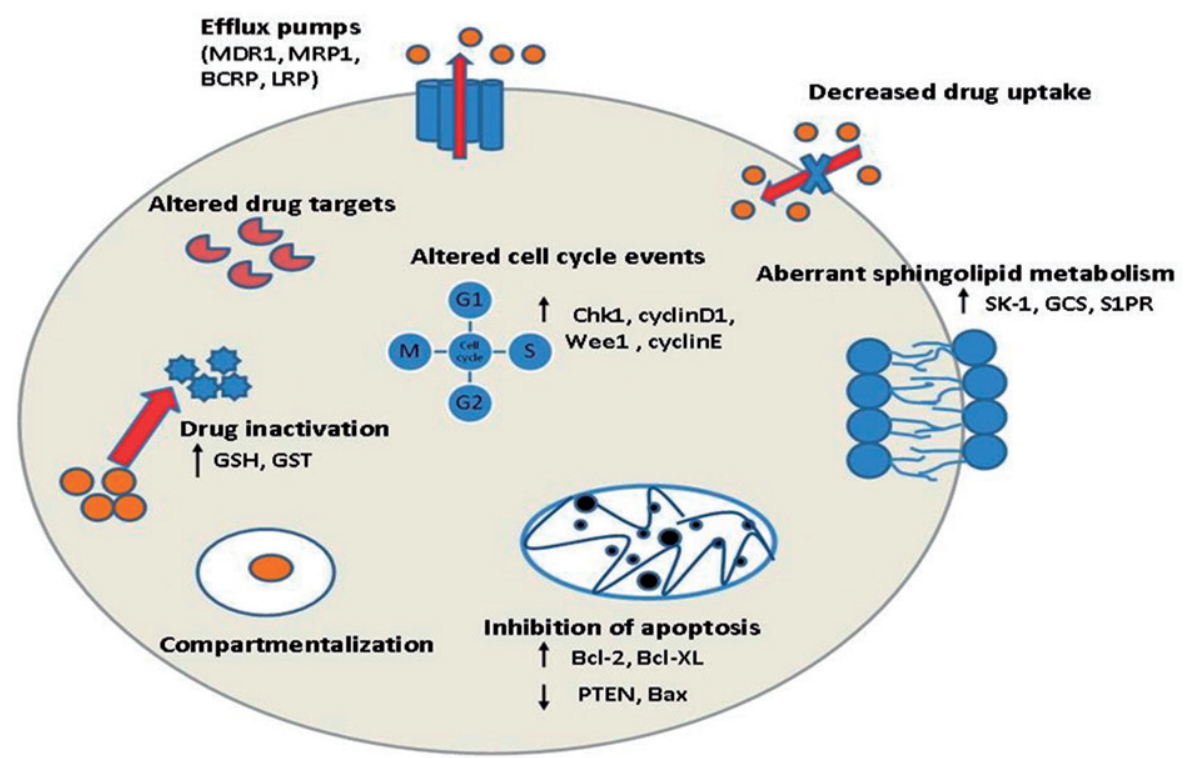

resistance mechanisms and touch on the approaches for reversing the resistance in cancer types.

\section{Decreased drug accumulation in cancer cells}

Expression of ATP-dependent efflux pumps are the most common mechanisms for the development of resistance against various drugs. ATP-dependent efflux pumps are the member of ATP-binding cassette (ABC) transporters family that have sequence and structural homology (Dean et al., 2001). These efflux pumps decrease intracellular drug concentrations and cause resistance. Vinca alkaloids, anthracyclines, actinomycin-D and paclitaxel are the examples of drugs that are affected by transporter-based MDR (Ambudkar et al., 1999).

\section{Pgp in anticancer drug resistance}

Among the $\mathrm{ABC}$ transporters involved in MDR, P-glycoprotein (Pgp) is the most common efflux pump in the plasma membrane with the molecular weight of $170 \mathrm{kDa}$ (Riordan \& Ling, 1979). Structurally, Pgp is a single polypeptide including two homologous parts each having a hydrophobic transmembrane domain (TMD) and a nucleotide-binding domain (NBD). These parts are separated by an intracellular linker region. Each TMD consists of six membrane spanning helices. Transmembrane domains are responsible for the specificity of substrate drugs by forming channels whereas nucleotide-binding domains participate in ATP binding and hydrolysis (Prajapati \& Sangamwar, 2014). Pgp is able to bind a large variety of hydrophobic drugs including most commonly used anticancer drugs such as doxorubicin, daunorubicin, vinblastine, vincristine and taxol, as well as many commonly used pharmaceuticals like antiarrhythmics and antihistamines (Bogman et al., 2001). The most common hypothesis related to Pgp-mediated transport suggests that the drug molecule binds to a specific site of Pgp and one of the ATP-binding domains is activated. Then, the hydrolysis of ATP causes a major change in the shape of Pgp, which causes the release of the drug into the extracellular space (Stavrovskaya, 2000). The MDR family includes two genes in man and only the ABCB1 (also called MDR1/Pgp) gene has been found to cause MDR. (Stavrovskaya, 2000). Pgp-related MDR can occur due to alteration of $\mathrm{ABCB} 1$ gene expression and amplification of the ABCB1 gene (Borst, 1991). The stabilization of ABCB1 mRNA, regulation at the level of synthesis and alterations of protein processing are other MDR mechanisms (Campos et al., 1992). Pgp has been found to be widely expressed in many human cancer types such as small and large intestine cancers, liver cancer, pancreatic cancer, myeloma, lymphoma, leukemia, kidney, ovary, testicle, neuroblastoma and fibrosarcoma (Goldstein et al., 1989) (Supplemental data 1 in Supplemental material).

One of the approaches to reverse MDR in cancer treatment is the inhibition of Pgp. There are three generations of Pgp inhibitors. The examples of first generation inhibitors are verapamil, quinine and cyclosporine $\mathrm{A}$. The second generation agents include dexverapamil and PSC833, and the inhibitors like XR9576 and LY335979 (Yan et al., 2012).

In addition to Pgp inhibitors, alternative approaches including peptides and antibodies to overcome MDR were also developed. Hydrophobic peptides such as reversins 121 and 205 are high-affinity Pgp substrates and inhibit Pgp (Sharom et al., 1999). Such peptides were specifically designed to target the transmembrane domain of Pgp and they were thought to prevent the assembly or function of Pgp (George et al., 2003). Recently, anti-Pgp monoclonal antibodies have been believed to be an alternative approach. One of these antibodies, such as UIC2, was designed to target extracellular epitopes of Pgp and inhibited in vitro efflux of drug substance (Mechetner \& Roninson, 1992). Moreover, it is known that the expression of the MDR gene is induced after cytotoxic drug treatment in tumor cells. Therefore, if MDR gene expression is inhibited by several approaches, MDR could be reversed. Using more specific techniques such as antisense oligonucleotides, hammerhead ribozymes and short-interfering RNA may provide more specific ways to cope with MDR (Liscovitch \& Lavie, 2002; Pichler et al., 
2005). Hao et al. (Hao et al., 2012) displayed that the ABCB1 gene was downregulated via RNA interference in human renal cell carcinoma and MDR resistance could be reversed. Downregulation of ABCB1 gene via specific siRNA reversed doxorubicin resistance in breast cancer cells and resulted in accumulation of the drug in the nuclei (Dönmez \& Gündüz, 2011). Recently, secondary metabolites such as phenolics, terpenoids and alkaloids were found to be potential Pgp inhibitors in colon and leukemia cells (Eid et al., 2013). In paclitaxel-resistant ovarian cancer cells with Pgp overexpression, a natural polyphenol was shown to overcome resistance by inhibiting the expression and function of Pgp through down-regulation of NF- $\mathrm{KB}$ activity and MAPK/ERK pathway (Zhao et al., 2013).

\section{MRP family in anticancer drug resistance}

Several studies have shown the presence of MDR in the absence of Pgp overexpression and indicated the role of other MDR related proteins. One of these proteins is encoded by MDR associated protein 1 (MRP1/ABCC1) which was found to be amplified in a non-P-gp MDR cells (Kruh \& Belinsky, 2003). ABCC1 protein, with molecular weight $\sim 190 \mathrm{kDa}$, includes three TMDs containing 17 membrane spanning helices, two NBDs and an extra N-terminal domain (Ozben, 2006). Like Pgp, MRP belongs to the family of ABC transporters and pumps toxic substances out of the cell in an ATP-dependent manner (Stavrovskaya, 2000). The drugs transported by $\mathrm{ABCC} 1$ are similar to Pgp substrates except taxanes which are poor substrates for $\mathrm{ABCC}$. Unlike $\mathrm{ABCB} 1, \mathrm{ABCC} 1$ can also export drugs modified by glycosylation, sulfation and glutathione (Ozben, 2006; Szakács et al., 2006). The other members of MRP family, MRP2/ABCC2, MRP3/ABCC3, MRP6/ABCC6 and MRP7/ $\mathrm{ABCC} 10$ have been displayed to have roles in resistance to anticancer agents (Borst et al., 2000). The transported drugs by $\mathrm{ABCC} 2$ are similar to $\mathrm{ABCC} 1$ only with few exceptions. For instance, cisplatin and camptothecin resistant cancer cells had higher ABCC2 levels (Annereau et al., 2004; Liedert et al., 2003). ABCC 3 exports fewer substrates compared to $\mathrm{ABCC} 1$ and $\mathrm{ABCC} 2$. In several cancer cells such as lung, acute myeloid leukemia, bladder and ovarian cancers, ABCC 3 expression has been observed (Kool et al., 1997). However, the data obtained from the literature indicated that among several members of MRP family, only $\mathrm{ABCC} 1$ has been commonly thought to be responsible for clinical drug resistance (Leonard et al., 2003). MRP-related resistance could be reversed by most of the Pgp inhibitors such as XR9576 (tariquidar), biricodar and isothiocyanates with the similar mechanisms of action whereas effective inhibitors of MRP members are also present. For example, genistein inhibited the daunorubicin uptake in MRP overexpressing cells but not in Pgp expressing cells (Sarkadi \& Müller, 1997). Lin et al. (Lin et al., 2013) displayed the drug reversal effects of MAPK pathway inhibitors, such as Raf1 and MEK inhibitors, in hepatocellular carcinoma and found that only MEK inhibitors reduced $\mathrm{ABCC} 1$ and $\mathrm{ABCC} 3$ expression and lead to increased drug accumulation in the cells.

$\mathrm{ABCC} 1$ is also expressed in a wide range of tumors and cancer cell lines including leukemia, pancreatic cancer and breast cancer (Szakács et al., 2004) (Supplemental data 2 in Supplemental material).

\section{BCRP in anticancer drug resistance}

The other $\mathrm{ABC}$ transporter involved in MDR is breast cancer resistance protein (BCRP/ABCG2) with molecular weight $\sim 75 \mathrm{kDa}$ which has the ability to transport methotrexate, 7-ethyl-10-hydroxycamptothecin or tyrosine kinase inhibitors (Szakács et al., 2006). In contrast to Pgp and MRP1, it contains only one NBD preceding one TMD with six membrane spanning helices ( $\mathrm{Ni}$ et al., 2010). BCRP has been identified to have a potential impact on drug resistance in hematologic malignancies like AML and CML due to its frequent expression on malignant hematopoietic and lymphoid cells (Natarajan et al., 2012). Although several BCRP substrates are transported out of the cell at low concentrations, they are also inhibitors of BCRP at higher concentrations. For instance, tyrosine kinase inhibitor, imatinib, inhibits BCRP function at higher concentrations (Houghton et al., 2004). Fumitremorgin C (FTC) analogues are specific inhibitors of BCRP and poloxamines and acrylonitrile derivatives have gained attention as potential inhibitors (Cuestas et al. 2011; Rabindran et al., 2000).

\section{LRP in anticancer drug resistance}

Lung resistance related protein or major vault protein (LRP/ MVP) with a molecular weight of $110 \mathrm{kDa}$ is not localized on the cell membrane like MDR1 and MRP1. LRP is found in the cytoplasm as a main component of multimeric vaults and associated with cytoskeletal elements as well as nuclear membrane, transporting drugs from nucleus to cytoplasm via vesicular trafficking (Stavrovskaya, 2000). There are some studies showing the relationship between drug resistance and LRP expression in AML and ovarian cancer patients (Chauhan et al., 2012; Zhao et al., 1999). In another study, LRP expression was significantly higher in colon carcinoma cells after sodium butyrate treatment and this caused resistance against adriamycin. LRP-mediated drug resistance could be reversed by a pyrimidine analog that could inhibit adriamycin efflux from nuclei (Kitazono et al., 2001). In a recent study, it has been found that magnetic $\mathrm{Fe}_{3} \mathrm{O}_{4}$ nanoparticles loaded with cisplatin could reverse cisplatin resistance in cisplatin-resistant lung cancer cells both in vitro and in vivo by decreasing the expression of LRP at both gene and protein levels ( $\mathrm{Li}$ et al., 2013). Src tyrosine kinase inhibition in cisplatin-resistant lung cancer cells by 4-anilinoquirazoline was found to downregulate LRP expression and sensitize resistant cells to cisplantin ( $\mathrm{Lv} \&$ Tian, 2012). Major MDR pumps involved in MDR and their significant properties mentioned in this part are summarized in Table 1.

\section{Decreased drug uptake}

Decreased drug uptake is another way to decrease drug accumulation in cancer cells. Drugs are transported into the cells via several routes which include diffusion across the plasma membrane, loading of the drugs on specific receptors and either receptor mediated or non-specific 
Table 1. Major MDR associated pumps: structure, localization, transported substrates, representative inhibitors and cancer types.

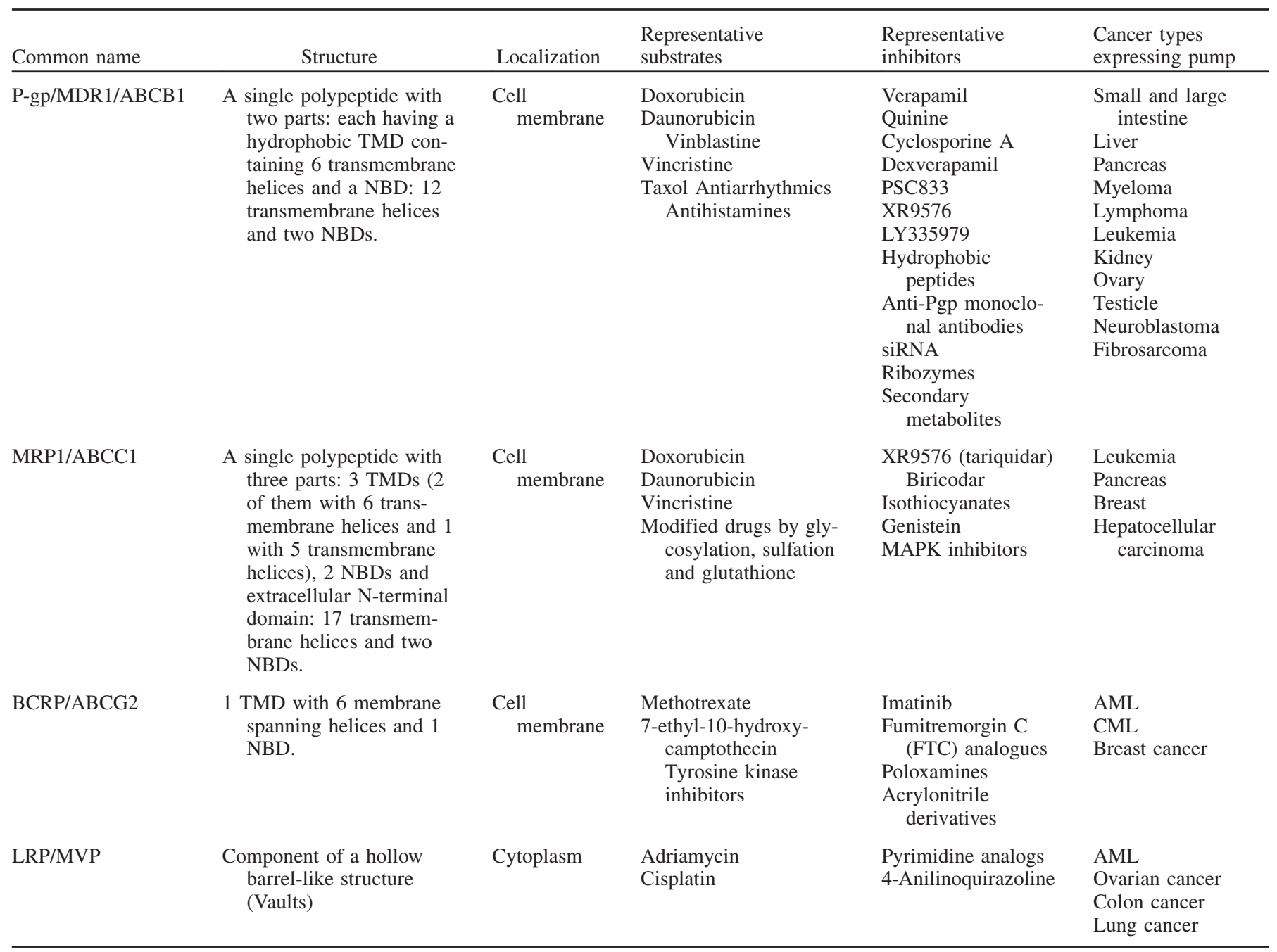

endocytosis (Gottesman, 2002). Development of drug resistance in some cancer cells could result from mutations that modify or block the receptors (Supplemental data 3 in Supplemental material). It is also known that endocytosis, and generally receptor-mediated endoyctosis, plays important roles in the transport of certain drugs into the cells and defective endocytic process causes drug resistance (Gottesman, 2002) (Supplemental data 3 in Supplemental material).

\section{Alterations in drug targets}

The cellular target of a particular cancer therapy could be altered or even decreased or increased to a level at which it may lose therapeutic potential. During anti-estrogen (e.g. tamoxifen) therapy of breast cancer, patients become resistant to endocrine therapy due to obvious loss of estrogen receptors in the resistant tumor cells that were believed not to be dependent on estrogen anymore for their growth (Luqmani, 2005). Sometimes, resistant cancer cells produce a mutated drug target that retains its activity in the cell without being a target of that drug. Therefore, the target is not inhibited by it (Supplemental data 4 in Supplemental material). Gene amplification is a marker of genomic instability in cancer cells and plays roles in the development of acquired drug resistance (Supplemental data 4 in Supplemental material).

\section{Altered cell cycle events}

An important feature of cancer cells is that uncontrolled cell proliferation arises from defects throughout the cell cycle progression at $\mathrm{G} 1, \mathrm{G} 2, \mathrm{~S}$ and mitotic phases. Cell cycle checkpoints, including a network of protein kinase signaling pathways, protect the cells from DNA damage induced by chemotherapeutic agents and provide the cells appropriate time to repair the damages (Sancar et al., 2004). Therefore, defects in cell cycle checkpoints could cause carcinogenesis and the development of drug resistance. Arrest of cell cycle progression at G2 phase generally gives the cells an opportunity to protect their viability after drug treatment and this arrest requires the activation of DNA damage checkpoint components such as Chk1, which is major checkpoint kinase (Hapke et al., 2001; Xiao et al., 2003). It was demonstrated that activation of the Chk1 kinase pathway could not only delay mitotic entry but also increase the potential of the cells to survive and lack of Chk1 in the cells could result in hypersensitivity to DNA damage inducing drugs (Walworth \& Bernards, 1996) (Supplemental data 5 in Supplemental material). Cell cycle-related drug resistance is most 
common in combination therapies in which the firstly given drug could affect the cell cycle and therefore, the next agent becomes less effective (Shah \& Schwartz, 2001) (Supplemental data 5 in Supplemental material).

\section{Compartmentalization}

Sequestration of the drugs in cellular compartments is an important mechanism for anticancer drug resistance. Although little is known about intracellular drug localization, an anticancer drug can whether pass through the cell membrane or not is an important fact in the localization in the cell. If a molecule cannot pass through the membrane, it is endocytosed and its localization is limited to lysosomes. This molecule can also be translocated into the cell by transporter molecules. As cellular compartments have different activities, they are organized with different features: each has a different $\mathrm{pH}$ in the lumen, different composition of lipids and also different proteins. All of these factors influence the localization of drugs in different cellular compartments (Saito et al., 2003). The susceptibility of a weak acid to a basic environment, or of a weak base to an acidic environment, known as $\mathrm{pH}$ partition, plays a key role in localization of a drug in different compartments. This partition results in the generation of an electrical charge and a decline in membrane permeability, and also results in drug accumulation (Duvvuri \& Krise, 2005).

Since drugs used in cancer therapy generally target molecules in the nucleus such as topoisomerases and DNA, and also the molecules in the other cellular organelles, the localization of an anticancer drug in the cell plays an important role in the effectivity of the drug (Hu \& Kavanagh, 2003) (Supplemental data 6 in Supplemental material).

In order to overcome this type of resistance, appropriate drugs should be produced, but there are a few studies on this area due to the difficulties in visualization of drug delivery in cultured cells (Duvvuri \& Krise, 2005).

\section{Alterations in membrane lipids}

In many types of cancer cells, there are differences in lipid profiles as compared to healthy cells (Leach, 1996). It was reported that in healthy and cancerous breast cells, phosphatidylserine, sphingomyelin, phosphatidylcholine, phosphatidylethanolamine and phosphatidylinositol levels are different (Merchant et al., 1991). Similarly, these phospholipids are found in distinctive levels in healthy and cancerous esophagus cells (Merchant et al., 1993). Merchant et al. also reported that cholesterol is found in different levels in the cells at different phases of colon cancer (Merchant et al., 1995). Interestingly, there was no difference in the phospholipid composition of healthy and cancerous liver cells, but cholesterol levels are found to be significantly lower in cancerous cells. By these changes in membrane lipid compositions, cancer cells make their membrane systems less permeable. Alterations in membrane lipids are important factors for acquiring multidrug resistance (Supplemental data 7 in Supplemental material).

In order to overcome this type of drug resistance, lipophilic cationic agents connecting with phospholipids found in the cell membrane could be used. By this way, membrane permeability could be increased. In a study, it was reported that calmodulin antagonists such as clomipramine and thioridazine, calcium channel blocker such as verapamil, beta adrenergic receptor blocker, propranolol and also antiparasitic agents like quinine are very effective whereas steroids and antineoplastic agents are not effective in influencing the membrane permeability (Castaing et al., 2000). Furthermore, bioactive sphingolipid levels are also altered in anticancer drug-resistant cancer cells (Ogretmen \& Hannun, 2004). The relation between the alterations in bioactive sphingolipid levels and the anticancer drug resistance will be discussed in the following sections.

\section{Sphingosine 1-phosphate in anticancer drug resistance}

Many studies indicated that increased ceramide levels lead to apoptosis whereas increased levels of sphingosine 1phosphate (S1P) inhibit apoptosis (Radin, 2002). In addition, this effect of S1P on cancer cells is a significant factor for developing anticancer drug resistance. In paclitaxel-resistant human ovarian cancer cells, paclitaxel treatment does not increase intracellular levels of ceramide, and instead, cellular GM3 ganglioside levels increase significantly as compared to sensitive cells (Prinetti et al., 2006). Furthermore, sphingosine kinase-1 (SK1-1), which is responsible for the synthesis of S1P, was reported to decrease the apoptotic effects of anticancer drugs in prostate adenocarcinoma cells (Pchejetski et al., 2005). In camptothecinresistant PC-3 prostate cancer cells, SK-1 expression levels were higher, and when this expression is suppressed, PC-3 cells became sensitive to camptothecin (Bektas et al., 2005). In addition, contributions of SK-1 to generation of drug resistance were also reported in many other types of cancer including kidney, breast, lung, uterus and colon (Visentin et al., 2006). Use of SK-1 inhibitor and S1P receptor antagonists could overcome anticancer drug resistance in these types of cancer cells (Ponnusamy et al., 2010) (Supplemental data 8 in Supplemental material).

\section{Glucosylceramide in anticancer drug resistance}

Glucosylceramide, which is synthesized from ceramide by the activity of glucosylceramide synthase (GCS), is also found to be increased in drug resistant cancer cells. In breast cancer cells, it was reported that suppression of GCS activity results in a decrease in MDR1 expression levels, and this reverses anticancer drug resistance (Gouazé et al., 2005). GCS and P-gp activity are known to be related in the generation of multidrug resistance phenotype in cancer cells (Ponnusamy et al., 2010).

Drug resistance arising from the overexpression of GCS can be reversed by inhibiting GCS activity, and also by increasing intracellular ceramide levels (Maurer et al., 2000). However, a study reported that suppression of GCS activity does not affect the sensitivity of melanoma cells against different types of anticancer agents (Veldman et al., 2003). Our studies also showed the effects of alterations in ceramide metabolism on drug resistance (Adan-Gokbulut et al. 2013; Kartal-Yandim et al., 2013) (Supplemental data 9 in Supplemental material). 


\section{Drug inactivation}

In this type of anticancer drug resistance mechanism, drugs become detoxified by the enzymatic activities of GST. Glutathione (GSH), a crucial antioxidant, prevents oxidative stress, and keeps redox homeostasis stable in cells (Gawryluk et al., 2011). In drug metabolism, GSH is used as a cofactor by GST enzyme system providing the formation of GSH-drug conjugates. In the cells resistant to alkylating agents such as cyclophosphamide, doxorubicin, melphalan and chlorambucil, it was found that GSH levels are higher than that of sensitive counterparts. In these cells, GSH forms conjugates with alkylating agents by the activity of GST, and this results in detoxification of alkylating agents, which causes drug resistance in cells (O'Brien \& Tew, 1996). Although the exact mechanism is not known yet, GSH synthesizing enzymes could also be important in the development of drug resistance. In addition, multidrug resistance in the cells overexpressing P-gp could also be related to the GSH system (Supplemental data 10 in Supplemental material).

To overcome this type of resistance, GST inhibitors and substrates for GSH conjugation are used. GSH conjugation substrates such as phorone and diethylmaleate immediately decrease the levels of GSH in many types of tissues in vivo (Mulder \& Ouwerkerk-Mahadevan, 1997). Buthionine sulfoximine (BSO) is also an effective and irreversible inhibitor for the synthesis of GSH. BSO, used in clinical studies in cancer, reverses resistance against alkylating agents via decreasing the levels of GSH in cells (Tew, 1994). In vitro studies indicated that BSO overcomes drug resistance arisen through MRP activity (Grant et al., 1994). Ethacrynic acid is also used in both in vitro and clinical trials in order to prevent GSH conjugation (Grant et al., 1994).

\section{Prevention of apoptosis}

Induction of apoptosis is known as an ultimate aim of anticancer agents. In apoptosis, DNA fragmentation, chromosome condensation and nuclear cleavage and finally cellular shrinkage occur, respectively. A network of genes and proteins are involved in the organization of apoptotic events or the maintenance of cell survival.

\section{p53 in anticancer drug resistance}

p53 is an important transcription factor with tumor suppressor functions (Wang et al., 2003). Except of the stress conditions, cells have inactive p53 protein with a short half-life at low levels. When the cell undergoes stress conditions such as DNA damage, hypoxia, nitric oxide exposure, decrease in ribonucleotides and oncogenic signaling, p53 becomes activated and intracellular levels of p53 proteins are increased (Pluquet \& Hainaut, 2001). After the activation, p53 prevents cellular proliferation via arresting cell cycle at G1 or G2 phase or via triggering apoptotic signals. In case of any damage on DNA, p53 induces cell cycle arrest and provides the cell an appropriate time needed for repairing the DNA (Smith \& Seo, 2002). When the damage is serious that cannot be repaired, p53, in turn, makes the cells undergo apoptosis (Wang et al., 2003).
Activation and inactivation of p53 is regulated by phosphorylation, ubiquitinylation, acetylation and interactions with other proteins (Bode \& Dong, 2004). Murine double minute 2 $(\mathrm{Mdm} 2)$ is the protein that majorly regulates p53 activity via protecting it from interacting with the transcription proteins through binding to the transcriptional activation domain of p53, and also via providing the ubiquitin ligase-mediated degradation of p53 (Honda et al., 1997).

In more than $60 \%$ of cancer types, abnormalities in p53 function were reported (Pirollo et al., 2000). These abnormalities lead to uncontrolled cell proliferation as DNA cannot be repaired and cell cycle cannot be arrested in the cells having mutated p53. Consequently, these abnormal p53 functions lead to development of anticancer drug resistance in cancer cells (Chang et al., 2000) (Supplemental data 11 in Supplemental material).

Drug resistance arisen from p53 function can be overcome by targeting p53 expression via chemoradiation for wild-type p53 activity, adenoviruses expressing mutant p53 for killing tumor cells, and also via using small molecules for wild type p53 activity. In addition, in order to activate p53, Mdm2 can also be targeted via using small molecules altering the binding of Mdm2 and p53 such as nutlins, benzodiazepinediones and inhibitors of Mdm2 (MI) made of spiro-oxindole, or via using inhibitors for E3 ubiquitin ligase activity of Mdm2 (Wang \& Sun, 2010). Nutlin-3 is a very specific antagonist for Mdm2p53 interaction. In retinoblastoma, osteosarcoma, breast cancer and colon cancer cells, p53 activity has been reported to be increased in response to nutlin-3 treatment (Laurie et al., 2006). However, nutlin-3 is a substrate for MRP1 and P-gp, which makes its use limited. It was reported that the application of nutlin-3 and curcumin in nanoparticles results in the modulation of efflux proteins by curcumin, and thus results in an increase in nutlin-3 function. The outcome of these events reverses multidrug resistance in Y79 human retinoblastoma cell lines (Das \& Sahoo, 2012). Benzodiazepinedione (BDA) is also a powerful molecule in recognizing the p53-binding domain of Mdm2 (Grasberger et al., 2005). It was reported that BDA treatment triggers p53 activity, suppresses cellular proliferation and also increases anticancer effects of doxorubicin synergistically both in vivo and in vitro (Koblish et al., 2006). Mdm2 inhibitors composed of spiro-oxindole compounds are the other molecules that bind to Mdm2. A type of these inhibitors reversed the etoposide resistance in A549 lung cancer cells, and led to apoptosis in response to the treatment (Sun et al., 2008).

\section{$\mathrm{Bcl}-2$ family genes in anticancer drug resistance}

$\mathrm{Bcl}-2$ is an oncogene which enhances cancer cell proliferation and suppresses apoptosis. In various types of cancer, Bcl-2 is expressed in an uncontrolled manner. The family of Bcl-2 also comprises the genes encoding proapoptotic Bax, Bad, Bim and antiapoptotic Bcl-XL and Bcl-2 proteins (Reed, 1995). Formation of homodimers and heterodimers between the members of this family determines the effect of these proteins on apoptosis. Heterodimers of Bcl-2 and Bax, and homodimers of bax trigger apoptosis whereas homodimers of Bcl-2 inhibit apoptosis (Reed, 1995) (Supplemental data 12 in Supplemental material). 
In order to overcome this type of resistance, agents targeting Bcl-2 family members for altering their expression levels are used commonly. It was shown that overexpression of miR-181b and miR-497 downregulate Bcl-2 and reverse drug resistance and induces apoptosis in vincristine-resistant SGC7901 human gastric cancer and cisplatin-resistant A549 human lung cancer cells (Zhu et al., 2010; Zhu et al., 2012). It was also demonstrated that targeting Bcl-2 by antisense peptide nucleic acid (PNA) conjugate decreases resistance and cellular proliferation in Mec-1 human non-Hodgkin's lymphoma cells (Balkin et al., 2011). In adriamycin-resistant H69AR human small cell lung cancer cells infected with Bcl-2 interfering RNA, there were decreases in the levels of $\mathrm{Bcl}-\mathrm{XL}$ and MRP1, in addition to Bcl-2, and also these cells were more sensitive to daunomycin treatment than that of sensitive counterparts (Palaniyandi et al., 2011). Moreover, inhibition of Bcl-2, Bcl-XL, XIAP and survivin via siRNAs reverses resistance against mitomycin $\mathrm{C}$ and cisplatin in EJ28 and J82 human bladder cancer cell lines (Kunze et al., 2012). There are also chemotherapeutic agents such as monocarboxylate transporter inhibitor $\alpha$-cyano-4-hydroxycinnamate $(\alpha \mathrm{CHC})$, cannabinoid receptor agonists, mebendazole (MBZ) and ABT-737 which act via decreasing intracellular Bcl-2 levels, and by this way resistant cancer cells become sensitized and undergo apoptosis (Doudican et al., 2013; Kumar et al., 2013; Rooswinkel et al. 2012; Xian et al., 2013).

\section{PTEN in anticancer drug resistance}

The phosphatase and tensin homolog, PTEN, is a tumor suppressor which has phosphatase activity preventing PI3K/ Akt signaling pathway, known as an important cancerpromoting pathway (Stambolic et al., 1998). In brain, endometrium, prostate and skin cancers, PTEN is generally found mutated, and also this mutated PTEN causes the development of anticancer drug resistance due to increased activation of PI3K/Akt signaling pathway (Ali et al., 1999).

The drug resistance arisen by PTEN mutation could be overcome via targeting PI3K/Akt signaling, and also via increasing intracellular levels of PTEN. In trastuzumabresistant SKBR3/R human breast cancer cell lines, the proteasome inhibitor PS341 treatment results in an increase in PTEN expression and induction of apoptosis due to increased chemosensitivity (Fujita et al., 2006). In BGC-823 human gastric cancer cell lines, PTEN upregulation via PEAK8-PTEN transfection sensitizes the cells against etoposide and doxorubicin treatment. In addition, use of a PI3K inhibitor, wortmannin, on BGC-823 and SGC-7901 human gastric cancer cell lines results in increased chemosensitization of the cells against etoposide and doxorubicin (Yu et al., 2008). Furthermore, PTEN transfection of PTEN-deficient PC3 human prostate cancer cell lines results in chemosensitization of the cells to doxorubicin and vinblastine through inhibiting the PI3K/Akt/mTOR signaling pathway (Sherbakova et al., 2008). In addition, recent studies have reported that suppression of autophagy could also sensitize PTEN-deficient PC3 human prostate cancer cells against Akt inhibitors like AZD5363 (Lamoureux et al., 2013). Another recent study has reported that microRNA- $19 \mathrm{a} / \mathrm{b}$ plays important roles in the regulation of drug resistance via targeting PTEN activity in gastric cancer cells (Wang et al., 2013).

\section{Clinical trials targeting MDR pathways}

We summarized selected phase studies of inhibitors/drug/ agents targeting reviewed MDR pathways in Table 2. Clinical trials of various first, second and third generation inhibitors,

Table 2. Selected phase studies of inhibitors/drug/agents targeting MDR pathways.

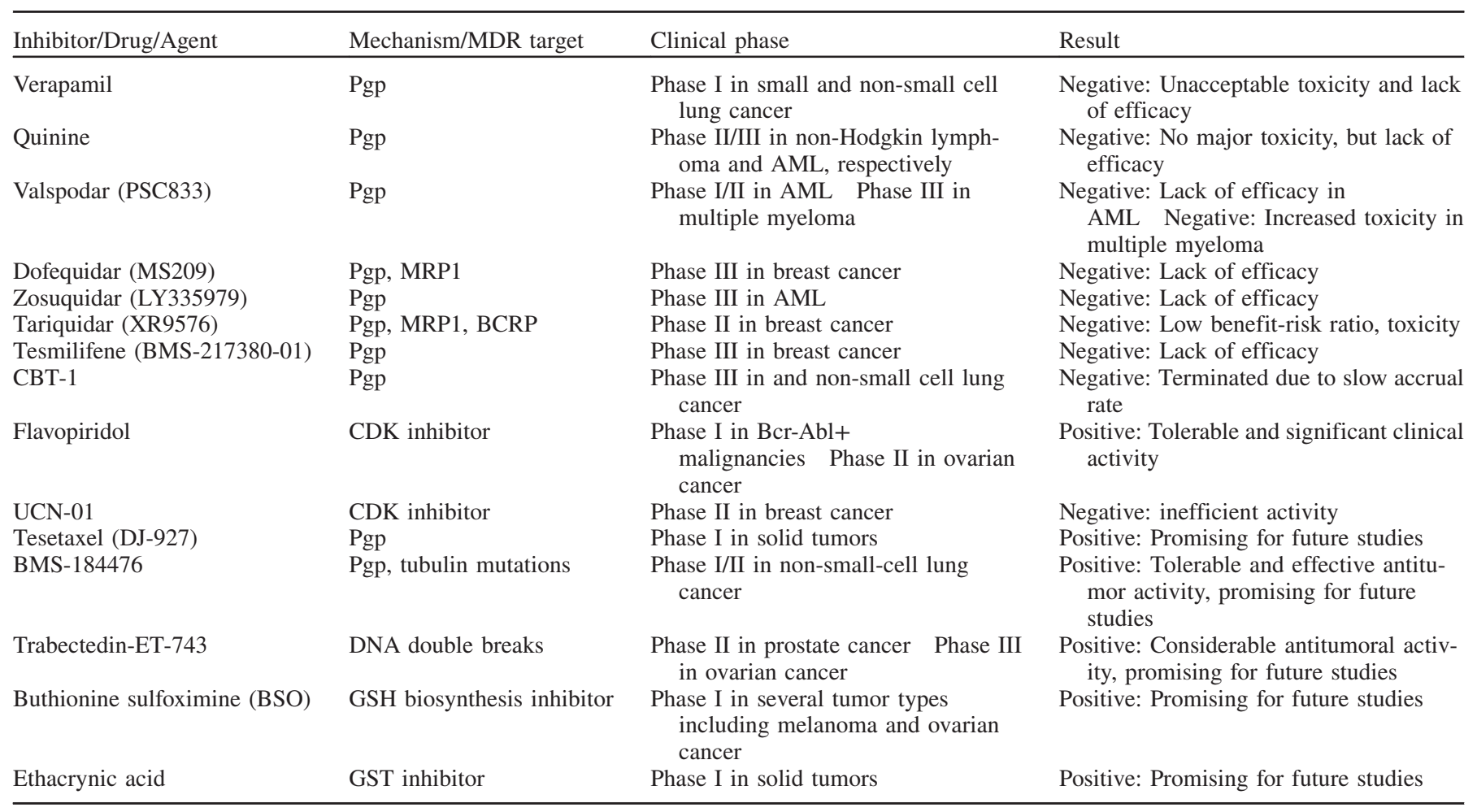


newly designed inhibitors targeting MDR transporters, agents targeting cell cycle regulators, GSH and GST and agents overcoming MDR by causing mutations and DNA double breaks were discussed in detail (see Supplemental data 13 in Supplemental material).

\section{Major obstacles to success in reversing clinical MDR}

Many MDR inhibitors have been entered into clinical trials, however they caused limited therapeutic success in the clinic due to the presence of several barriers. A common reason for early termination or clinical failure of MDR modulators is their non-specific toxicity to cancer patients. This situation is particularly true for first generation Pgp inhibitors that cause unacceptable toxicity at doses required for P-gp inhibition (Millward et al., 1993). While these effects are less for second and third generation inhibitors, they inhibit $\mathrm{ABC}$ transporters in normal tissues as well, which can lead to toxicity (Yu et al., 2013). Another common obstacle is the unexpected and undesired pharmacokinetic interactions between the modulators and the anti-cancer drugs used for the treatment of patients, which results in reducing doses of anticancer drugs and so inefficient benefit (Patel \& Mitra, 2001). The multifactorial nature of MDR is another obstacle, causing inefficient therapy of patients. As some reasons for MDR could not be related to ABC transporters or different transporters could be responsible for removal of an anticancer drug (Li et al., 2009).

Recently, the probability of drug-transporter meeting has been defined as a significant limiting factor in MDR. As dictated in MDR definition, the cells are resistant to many drugs that are not necessarily chemically related. This situation is not only related to specificity between a drug and a transporter, but also related to increased drugtransporter meeting by changing the spatial organization of membrane (Panagiotopoulou et al., 2010). The increased affinity between drug and transporter in MDR can be explained physically by the mechanical interaction among the drug size (MW), membrane endocytosis and drug transporter surface density and functionality. For instance, the MW of drugs is involved in their residency time in membrane and function of the membrane physical properties. Moreover, the understanding of drug pumping kinetics will allow researchers to consider that a drug can meet a transporter, but this transporter is in an occupied state while pumping another drug. To overcome MDR, new therapeutic strategies could be designed based on this knowledge (Daniel et al., 2013). In MDR cells, cytosolic pH becomes alkalic by the activity of the proton pump vacuolar-type ATPase and the proton transporters, which somehow affects the packing of lipids, thereby influencing the transverse movement of drugs. One hypothesis explains that alkalic $\mathrm{pH}$ can affect negatively charged lipids in the inner leaflet of the membrane, affecting repulsion between lipids. This alkaline $\mathrm{pH}$ results in the failure of the accumulation of chemotherapeutic agents together with increased drug efflux (Simon et al., 1994). Thus, MDR could be prevented by targeting both $\mathrm{pH}$ changes and transporters. Several properties of membrane such as the fluidity and lipid density are also interested in MDR. For instance, an excess of packing of lipid in the inner membrane of MDR cells is responsible for blocking drugs (Rauch, 2009). Resistant cells also have faster endocytosis as compared to sensitive counterparts, which is related to higher levels of endogenous compression of the inner leaflet of the membrane (driving force for endocytosis) (Rauch \& Pluen, 2007). In conclusion, the introduction of $\mathrm{pH}$ concept in MDR and the understanding of physical properties of membrane will lead to development of "new synthetic theories" to understand MDR as a whole and to develop future treatment strategies for MDR.

\section{Conclusion and future perspectives}

Studies aiming to investigate the molecular mechanisms of multidrug resistance obviously show that there are many factors leading to anticancer drug resistance. We have described most effective mechanisms in this review.

Targeting vital genes encoding membrane transporters or the other proteins that have important contributions to the generation of multidrug resistance phenotype by knockdown approaches or using chemical agents has a significant potential in clinic. These approaches would provide the reversal of the resistance, and cancer patients could be completely cured. We have also discussed clinical phase studies of described agents and their conclusions in this review. However, clinical approaches need more in vivo research studies, and also more extensive clinical phase trials on cancer patients. More importantly, inventions of new effective drug targeting and delivery systems that cause minimal adverse effects are needed. By these approaches, only multidrug resistant cancer cells rather than healthy cells could be targeted and also eradicated.

\section{Declaration of interest}

None of the authors have any interests, which might influence the compilation of the current literature in this subject. We apologize to the authors whose valuable studies were not included here due to space limitations and the concentrated scope of the review.

\section{References}

Adan-Gokbulut A, Kartal-Yandim M, Iskender G, Baran Y. (2013). Novel agents targeting bioactive sphingolipids for the treatment of cancer. Curr Med Chem, 20, 108-22.

Ali IU, Schriml LM, Dean M. (1999). Mutational spectra of PTEN/ MMAC1 gene: a tumor suppressor with lipid phosphatase activity. J Natl Cancer Inst, 91, 1922-32.

Ambudkar SV, Dey S, Hrycyna CA, et al. (1999). Biochemical, cellular, and pharmacological aspects of the multidrug transporter. Annu Rev Pharmacol Toxicol, 39, 361-98.

Annereau JP, Szakács G, Tucker CJ, et al. (2004). Analysis of ATPbinding cassette transporter expression in drug-selected cell lines by a microarray dedicated to multidrug resistance. Mol Pharmacol, 66, 1397-405.

Balkin ER, Jia F, Miller WH, Lewis MR. (2011). In vitro evaluation of targeted antisense 177Lu radiotherapy. Anticancer Res, 31, 3143-9.

Barker CJ, Bowler K. (1991). Lipid composition of the membranes from cells of two rat tumors and its relationship to tumor thermosensitivity. Radiat Res, 125, 48-55.

Bektas M, Jolly PS, Müller C, et al. (2005). Sphingosine kinase activity counteracts ceramide-mediated cell death in human melanoma cells: role of Bcl-2 expression. Oncogene, 24, 178-87. 
Bode AM, Dong Z. (2004). Post-translational modification of p53 in tumorigenesis. Nat Rev Cancer, 4, 793-805.

Bogman K, Peyer AK, Török M, et al. (2001). HMG-CoAreductase inhibitors and P-glycoprotein modulation. Br J Pharmacol, 132, 1183-92.

Borst P. (1991). Genetic mechanisms of drug resistance: a review. Acta Oncol, 30, 87-105.

Borst P, Evers R, Kool M, Wijnholds J. (2000). A family of drug transporters: the multidrug resistance-associated proteins. J Natl Cancer Inst, 92, 1295-301.

Campos L, Guyotat D, Archimbaud E, et al. (1992). Clinical significance of multidrug resistance P-glycoprotein expression on acute nonlymphoblastic leukemia cells at diagnosis. Blood, 79, 473-6.

Castaing M, Brouant P, Loiseau A, et al. (2000). Membrane permeation by multidrug-resistance-modulators and non-modulators: effects of hydrophobicity and electric charge. J Pharm Pharmacol, 52, 289-96.

Chang EH, Pirollo KF, Bouker KB. (2000). Tp53 gene therapy: a key to modulating resistance to anticancer therapies? Mol Med Today, 6, 358-64.

Chauhan PS, Bhushan B, Singh LC, et al. (2012). Expression of genes related to multiple drug resistance and apoptosis in acute leukemia: response to induction chemotherapy. Exp Mol Pathol, 92, 44-9.

Choi $\mathrm{CH}$. (2005). ABC transporters as multidrug resistance mechanisms and the development of chemosensitizers for their reversal. Cancer Cell Int, 30, 1-13.

Cuestas ML, Sosnik A, Mathet V. (2011). Poloxamines display a multiple inhibitory activity of ATP-Binding Cassette (ABC) transporters in cancer cell lines. Mol Pharm, 8, 1152-64.

Daniel C, Bell C, Burton C, et al. (2013). The role of proton dynamics in the development and maintenance of multidrug resistance in cancer. Biochimica et Biophysica Acta, 1832, 606-17.

Das M, Sahoo SK. (2012). Folate decorated dual drug loaded nanoparticle: role of curcumin in enhancing therapeutic potential of nutlin-3a by reversing multidrug resistance. PLoS One, 7, e32920.

Dean M, Rzhetsky A, Alliknets R. (2001). The human ATP-binding cassette (ABC) transporter superfamily. Genome Res, 11, 1156-66.

Demant EJ, Sehested M, Jensen PB. (1990). A model for computer simulation of P-glycoprotein and transmembrane delta $\mathrm{pH}$-mediated transport in multidrug-resistant tumor cells. Biochim Biophys Acta, $1055,117-25$.

Dönmez Y, Gündüz U. (2011). Reversal of multidrug resistance by small interfering RNA (siRNA) in doxorubicin-resistant MCF-7 breast cancer cells. Biomed Pharmacother, 65, 85-9.

Doudican NA, Byron SA, Pollock PM, Orlow SJ. (2013). XIAP downregulation accompanies mebendazole growth inhibition in melanoma xenografts. Anticancer Drugs, 24, 181-8.

Duvvuri M, Krise JP. (2005). Intracellular drug sequestration events associated with the emergence of multidrug resistance: a mechanistic review. Front Biosci, 10, 1499-509.

Eid SY, El-Readi MZ, Eldin EE, et al. (2013). Influence of combinations of digitonin with selected phenolics, terpenoids, and alkaloids on the expression and activity of P-glycoprotein in leukaemia and colon cancer cells. Phytomedicine, 21, 47-61.

Fujita T, Doihara H, Washio K, et al. (2006). Proteasome inhibitor bortezomib increases PTEN expression and enhances trastuzumabinduced growth inhibition in trastuzumab-resistant cells. Anticancer Drugs, 17, 455-62.

Gawryluk JW, Wang JF, Andreazza AC, et al. (2011). Decreased levels of glutathione, the major brain antioxidant, in post-mortem prefrontal cortex from patients with psychiatric disorders. Int $J$ Neuropsychopharmacol, 14, 123-30.

George SR, Ng GY, Lee SP, et al. (2003). Blockade of G protein-coupled receptors and the dopamine transporter by a transmembrane domain peptide: novel strategy for functional inhibition of membrane proteins in vivo. J Pharmacol Exp Ther, 307, 481-9.

Giaccone G, Pinedo HM. (1996). Drug resistance. Oncologist, 1, 82-7. Goldstein LJ, Galski H, Fojo A, et al. (1989). Expression of a multidrug resistance gene in human cancers. J Natl Cancer Inst, 81, 116-24.

Gottesman MM. (2002). Mechanisms of cancer drug resistance. Ann Rev Med, 53, 615-27.

Gouazé V, Liu YY, Prickett CS, et al. (2005). Glucosylceramide synthase blockade down-regulates P-glycoprotein and resensitizes multidrug- resistant breast cancer cells to anticancer drugs. Cancer Res, 65, 3861-7.

Grant CE, Valdimarsson G, Hipfner DR, et al. (1994). Overexpression of multidrug resistance-associated protein (MRP) increases resistance to natural product drugs. Cancer Res, 54, 357-61.

Grasberger BL, Lu T, Schubert C, et al. (2005). Discovery and cocrystal structure of benzodiazepinedione HDM2 antagonists that activate p53 in cells. J Med Chem, 48, 909-12.

Hao YX, He ZW, Zhu JH, et al. (2012). Reversal of multidrug resistance in renal cell carcinoma by short hairpin RNA targeting MDR1 gene. Chin Med J (Engl), 125, 2741-5.

Hapke G, Yin MB, Rustum YM. (2001). Targeting molecular signals in chk1 pathways as a new approach for overcoming drug resistance. Cancer Metastasis Rev, 20, 109-15.

Honda R, Tanaka H, Yasuda H. (1997). Oncoprotein MDM2 is a ubiquitin ligase E3 for tumor suppressor p53. FEBS Lett, 420, 25-7.

Houghton PJ, Germain GS, Harwood FC, et al. (2004). Imatinib mesylate is a potent inhibitor of the ABCG2 (BCRP) transporter and reverses resistance to topotecan and $\mathrm{SN}-38$ in vitro. Cancer Res, 64, 2333-7.

$\mathrm{Hu}$ W, Kavanagh JJ. (2003). Anticancer therapy targeting the apoptotic pathway. Lancet Oncol, 4, 721-9.

Kartal-Yandim M, Apohan E, Baran Y. (2013). Therapeutic potential of targeting ceramide/glucosylceramide pathway in cancer. Cancer Chemother Pharmacol, 71, 13-20.

Kitazono M, Okumura H, Ikeda R, et al. (2001). Reversal of LRPassociated drug resistance in colon carcinoma SW-620 cells. Int J Cancer, 91, 126-31.

Koblish HK, Zhao S, Franks CF, et al. (2006). Benzodiazepinedione inhibitors of the Hdm2:p53 complex suppress human tumor cell proliferation in vitro and sensitize tumors to doxorubicin in vivo. Mol Cancer Ther, 5, 160-9.

Kool M, de Haas M, Scheffer GL, et al. (1997). Analysis of expression of cMOAT (MRP2), MRP3, MRP4, and MRP5, homologues of the multidrug resistance-associated protein gene (MRP1), in human cancer cell lines. Cancer Res, 57, 3537-47.

Krishna R, Mayer LD. (2000). Multidrug resistance (MDR) in cancer mechanisms, reversal using modulators of MDR and the role of MDR modulators in influencing the pharmacokinetics of anticancer drugs. Eur J Pharm Sci, 11, 265-83.

Kruh GD, Belinsky MG. (2003). The MRP family of drug efflux pumps. Oncogene, 22, 7537-52.

Kumar A, Kant S, Singh SM. (2013). Targeting monocarboxylate transporter by $\alpha$-cyano-4-hydroxycinnamate modulates apoptosis and cisplatin resistance of Colo205 cells: implication of altered cell survival regulation. Apoptosis, 18, 1574-85.

Kunze D, Erdmann K, Froehner M, et al. (2012). siRNA-mediated inhibition of antiapoptotic genes enhances chemotherapy efficacy in bladder cancer cells. Anticancer Res, 32, 4313-18.

Lamoureux F, Thomas C, Crafter C, et al. (2013). Blocked autophagy using lysosomotropic agents sensitizes resistant prostate tumor cells to the novel Akt inhibitor AZD5363. Clin Cancer Res, 19, 833-44.

Larsen AK, Escargueil AE, Skladanowski A. (2000). Resistance mechanisms associated with altered intracellular distribution of anticancer agents. Pharmacol Ther, 85, 217-29.

Larsen AK, Skladanowski A. (1998). Cellular resistance to topoisomerase-targeted drugs: from drug uptake to cell death. Biochem Biophys Acta, 1400, 257-74.

Laurie NA, Donovan SL, Shih CS, et al. (2006). Inactivation of the p53 pathway in retinoblastoma. Nature, 444, 61-6.

Leach MO. (1996). Introduction to in vivo MRS of cancer: new perspectives and open problems. Anticancer Res, 16, 1503-14.

Leonard GD, Fojo T, Bates SE. (2003). The role of ABC transporters in clinical practice. Oncologist, 8, 411-24.

Li J, Li ZN, Du YJ, et al. (2009). Expression of MRP1, BCRP, LRP, and ERCC1 in advanced non-small-cell lung cancer: correlation with response to chemotherapy and survival. Clin Lung Cancer, 10, 414-21.

Li K, Chen B, Xu L, et al. (2013). Reversal of multidrug resistance by cisplatin-loaded magnetic $\mathrm{Fe}_{3} \mathrm{O}_{4}$ nanoparticles in A549/DDP lung cancer cells in vitro and in vivo. Int J Nanomedicine, 8, 1867-77.

Liedert B, Materna V, Schadendorf D, et al. (2003). Overexpression of cMOAT (MRP2/ABCC2) is associated with decreased formation of 
platinum- DNA adducts and decreased G2-arrest in melano.ma cells resistant to cisplatin. J Invest Dermatol, 121, 172-6.

Lin S, Hoffmann K, Xiao Z, et al. (2013). MEK inhibition induced downregulation of MRP1 and MRP3 expression in experimental hepatocellular carcinoma. Cancer Cell Int, 13, 1-10.

Liscovitch M, Lavie Y. (2002). Cancer multidrug resistance: a review of recent drug discovery research. IDrugs, 5, 349-55.

Luqmani YA. (2005). Mechanisms of drug resistance in cancer chemotherapy. Med Princ Pract, 14, 35-48.

Lv J, Tian Y. (2012). Effect of Src tyrosine kinase inhibition on the drugresistance as well as MDR1 and LRP expression of the human cisplatinum-resistant lung cancer cell line A549/DDP. Zhongguo Fei Ai Za Zhi, 15, 501-6.

Maurer BJ, Melton L, Billups C, et al. (2000). Synergistic cytotoxicity in solid tumor cell lines between $\mathrm{N}$-(4-hydroxyphenyl)retinamide and modulators of ceramide metabolism. J Natl Cancer Inst, 92, 1897-909.

Mechetner EB, Roninson IB. (1992). Efficient inhibition of P-glycoprotein-mediated multidrug resistance with a monoclonal antibody. Proc Natl Acad Sci USA, 89, 5824-8.

Merchant TE, Meneses P, Gierke LW, et al. (1991). 31P magnetic resonance phospholipid profiles of neoplastic human breast tissues. Br J Cancer, 63, 693-8.

Merchant TE, de Graaf PW, Minsky BD, et al. (1993). Esophageal cancer phospholipid characterization by 31P NMR. NMR Biomed, 6, 187-93.

Merchant TE, Diamantis PM, Lauwers G, et al. (1995). Characterization of malignant colon tumors with $31 \mathrm{P}$ nuclear magnetic resonance phospholipid and phosphatic metabolite profiles. Cancer, 76, 1715-23.

Millward MJ, Cantwell BM, Munro NC, et al. (1993). Oral verapamil with chemotherapy for advanced non-small cell lung cancer: a randomised study. Br J Cancer, 67, 1031-5.

Mulder GJ, Ouwerkerk-Mahadevan S. (1997). Modulation of glutathione conjugation in vivo: how to decrease glutathione conjugation in vivo or in intact cellular systems in vitro. Chem Biol Interact, 105, $17-34$.

Natarajan K, Xie Y, Baer RMT, Ross DR. (2012). Role of breast cancer resistance protein (BCRP/ABCG2) in cancer drug resistance. Biochem Pharmacol, 83, 1084-103.

Ni Z, Bikadi Z, Rosenberg MF, Mao Q. (2010). Structure and function of the human breast cancer resistance protein (BCRP/ABCG2). Curr Drug Metab, 11, 603-17.

O'Brien ML, Tew KD. (1996). Glutathione and related enzymes in multidrug resistance. Eur J Cancer, 32, 967-78.

Ogretmen B, Hannun YA. (2004). Biologically active sphingolipids in cancer pathogenesis and treatment. Nat Rev Cancer, 4, 604-16.

Ozben T. (2006). Mechanisms and strategies to overcome multiple drug resistance in cancer. FEBS Lett, 580, 2903-9.

Palaniyandi K, Zhao Q, Chang XB. (2011). Infection of H69AR cells with retroviral particles harboring interfering RNAi significantly reduced the multidrug resistance of these small cell lung cancer cells. Int J Biochem Mol Biol, 2, 155-67.

Panagiotopoulou V, Richardson G, Jensen OE, Rauch C. (2010). On a biophysical and mathematical model of Pgp-mediated multidrug resistance: understanding the "space-time" dimension of MDR. Eur Biophys J, 39, 201-11.

Patel J, Mitra AK. (2001). Strategies to overcome simultaneous P-glycoprotein mediated efflux and CYP3A4 mediated metabolism of drugs. Pharmacogenomics, 2, 401-15.

Pchejetski D, Golzio M, Bonhoure E, et al. (2005). Sphingosine kinase-1 as a chemotherapy sensor in prostate adenocarcinoma cell and mouse models. Cancer Res, 65, 11667-75.

Pichler A, Zelcer N, Prior JL, et al. (2005). In vivo RNA interferencemediated ablation of MDR1 P-glycoprotein. Clin Cancer Res, 11, 4487-94.

Pirollo KF, Bouker KB, Chang EH. (2000). Does p53 status influence tumor response to anticancer therapies? Anticancer Drugs, 11, 419-32.

Pluquet O, Hainaut P. (2001). Genotoxic and non-genotoxic pathways of p53 induction. Cancer Lett, 174, 1-15.

Ponnusamy S, Meyers-Needham M, Senkal CE, et al. (2010). Sphingolipids and cancer: ceramide and sphingosine-1-phosphate in the regulation of cell death and drug resistance. Future Oncol, 6, 1603-24.
Prajapati R, Sangamwar AT. (2014). Translocation mechanism of Pglycoprotein and conformational changes occurring at drug-binding site: insights from multi-targeted molecular dynamics. Biochim Biophys Acta, 1838, 2882-98.

Prinetti A, Millimaggi D, D’Ascenzo S, et al. (2006). Lack of ceramide generation and altered sphingolipid composition are associated with drug resistance in human ovarian carcinoma cells. Biochem J, 395, 311-18.

Rabindran SK, Ross DD, Doyle LA, et al. (2000). Fumitremorgin C reverses multidrug resistance in cells transfected with the breast cancer resistance protein. Cancer Res, 60, 47-50.

Radin NS. (2002). The development of aggressive cancer: a possible role for sphingolipids. Cancer Invest, 20, 779-86.

Rauch C. (2009). Toward a mechanical control of drug delivery. On the relationship between Lipinski's 2nd rule and cytosolic $\mathrm{pH}$ changes in doxorubicin resistance levels in cancer cells: a comparison to published data, Eur Biophys J, 38, 829-46.

Rauch C, Pluen A. (2007). Multi drug resistance-dependent "vacuum cleaner" functionality potentially driven by the interactions between endocytosis, drug size and Pgp-like transporters surface density, Eur Biophys J, 36, 121-31.

Reed JC. (1995). Bcl-2: prevention of apoptosis as a mechanism of drug resistance. Hematol Oncol Clin North Am, 9, 451-73.

Riordan JR, Ling V. (1979). Purification of P-glycoprotein from plasma membrane vesicles of Chinese hamster ovary cell mutants with reduced colchicine permeability. J Biol Chem, 254, 12701-5.

Rooswinkel RW, van de Kooij B, Verheij M, Borst J. (2012). Bcl-2 is a better ABT-737 target than Bcl-xL or Bcl-w and only Noxa overcomes resistance mediated by Mcl-1, Bfl-1, or Bcl-B. Cell Death Dis, 3, e366.

Saito G, Swanson JA, Lee KD. (2003). Drug delivery strategy utilizing conjugation via reversible disulfide linkages: role and site of cellular reducing activities. Adv Drug Deliv Rev, 55, 199-215.

Sancar A, Lindsey-Boltz LA, Unsal-Kacmaz K, Linn S. (2004) Molecular mechanisms of mammalian DNA repair and the DNA damage checkpoints. Annu Rev Biochem, 73, 39-85.

Sarkadi B, Müller M. (1997). Search for specific inhibitors of multidrug resistance in cancer. Sem Cancer Biol, 8, 171-82.

Schisselbauer JC, Silber R, Papadopoulos E, et al. (1990). Characterization of glutathione S-transferase expression in lymphocytes from chronic lymphocytic leukemia patients. Cancer Res, 50, 3562-68

Shah MA, Schwartz GK. (2001). Cell cycle-mediated drug resistance: an emerging concept in cancer therapy. Clin Cancer Res, 7, 2168-81.

Sharom FJ, Yu X, Lu P, et al. (1999). Interaction of the P-glycoprotein multidrug transporter (MDR1) with high affinity peptide chemosensitizers in isolated membranes, reconstituted systems, and intact cells. Biochem Pharmacol, 58, 571-86.

Sherbakova EA, Stromskaia TP, Rybalkina EIU, et al. (2008). Role of PTEN protein in multidrug resistance of prostate cancer cells. Mol Biol, 42, 487-93.

Simon S, Roy D, Schindler M. (1994). Intracellular pH and the control of multidrugresistance. Proc Natl Acad Sci USA, 91, 1128-32.

Smith ML, Seo YR. (2002). p53 regulation of DNA excision repair pathways. Mutagenesis, 17, 149-56.

Stambolic V, Suzuki A, de la Pompa JL, et al. (1998). Negative regulation of $\mathrm{PKB} / \mathrm{Akt}$-dependent cell survival by the tumor suppressor PTEN. Cell, 95, 29-39.

Stavrovskaya AA. (2000). Cellular mechanisms of multidrug resistance of tumor cells. Biochemistry, 65, 95-106.

Sun SH, Zheng M, Ding K, et al. (2008). A small molecule that disrupts Mdm2-p53 binding activates p53, induces apoptosis, and sensitizes lung cancer cells to chemotherapy. Cancer Biol Ther, 7, $845-52$.

Szakács G, Paterson JK, Ludwig JA, et al. (2006). Targeting multidrug resistance in cancer. Nat Rev, 3, 219-34.

Szakács G, Annereau JP, Lababidi S, et al. (2004). Predicting drug sensitivity and resistance: profiling $\mathrm{ABC}$ transporter genes in cancer cells. Cancer Cell, 6, 129-37.

Tew KD. (1994). Glutathione-associated enzymes in anticancer drug resistance. Cancer Res, 54, 4313-20.

Veldman RJ, Mita A, Cuvillier O, et al. (2003). The absence of functional glucosylceramide synthase does not sensitize melanoma cells for anticancer drugs. FASEB J, 17, 1144-6. 
Visentin B, Vekich JA, Sibbald BJ, et al. (2006). Validation of an antisphingosine-1-phosphate antibody as a potential therapeutic in reducing growth, invasion, and angiogenesis in multiple tumor lineages. Cancer Cell, 9, 225-38.

Walworth NC, Bernards R. (1996). Rad-dependent response of the chk1encoded protein kinase at the DNA damage checkpoint. Science, 271, 353-6.

Wang F, Li T, Zhang B, et al. (2013). MicroRNA-19a/b regulates multidrug resistance in human gastric cancer cells by targeting PTEN. Biochem Biophys Res Commun, 434, 688-94.

Wang W, Rastinejad F, El-Deiry WS. (2003). Restoring p53-dependent tumor suppression. Cancer Biol Ther, 2, S55-63.

Wang Z, Sun Y. (2010). Targeting p53 for novel anticancer therapy. Transl Oncol, 3, 1-12.

Xian XS, Park H, Choi MG, Park JM. (2013). Cannabinoid receptor agonist as an alternative drug in 5-fluorouracil-resistant gastric cancer cells. Anticancer Res, 33, 2541-7.

Xiao Z, Chen Z, Gunasekera AH, et al. (2003). Chk1 mediates S and G2 arrests through Cdc25A degradation in response to DNA-damaging agents. J Biol Chem, 278, 21767-73.

Yan XU, Feng ZHI, Guangming XU, et al. (2012). Overcoming multidrug-resistance in vitro and in vivo using the novel P-glycoprotein inhibitor 1416. Biosci Rep, 32, 559-66.
Yu HG, Ai YW, Yu LL, et al. (2008). Phosphoinositide 3-kinase/Akt pathway plays an important role in chemoresistance of gastric cancer cells against etoposide and doxorubicin induced cell death. Int $\mathbf{J}$ Cancer, 122, 433-43.

Yu M, Ocana A, Tannock IF. (2013). Reversal of ATP-binding cassette drug transporter activity to modulate chemoresistance: why has it failed to provide clinical benefit? Cancer Metastasis Rev, 32, 211-27.

Zhao BX, Sun YB, Wang SQ, et al. (2013). Grape seed procyanidin reversal of P-glycoprotein associated multi-drug resistance via downregulation of NF- $\mathrm{kb}$ and MAPK/ERK mediated YB-1 activity in A2780/T cells. PLoS One, 8, e71071.

Zhao Y, Yu L, Lou F, et al. (1999). The clinical significance of lung resistance-related protein gene (lrp), multidrug resistance-associated protein gene (mrp) and mdr-1/p170 expression in acute leukemia. Zhonghua Nei Ke Za Zhi, 38, 760-3.

Zhu W, Shan X, Wang T, et al. (2010). miR-181b modulates multidrug resistance by targeting BCL2 in human cancer cell lines. Int J Cancer, 127, 2520-9.

Zhu W, Zhu D, Lu S, et al. (2012). miR-497 modulates multidrug resistance of human cancer cell lines by targeting BCL2. Med Oncol, $29,384-91$.

Supplementary material available online

Supplementary Data 1-13 